\title{
HYBRID STARS THAT MASQUERADE AS NEUTRON STARS
}

\author{
Mark Alford and Matt Braby \\ Physics Department, Washington University, Compton Hall, 1 Brookings Drive, Campus Box 1105, St. Louis, MO 63130 \\ MARK PARIS ${ }^{1}$ \\ Jefferson National Laboratory, 12000 Jefferson Avenue, Newport News, VA 23606 \\ AND \\ SANJAY REDDY \\ Theoretical Division, Los Alamos National Laboratory, Los Alamos, NM 87545 \\ Received 2005 January 5; accepted 2005 April 8
}

\begin{abstract}
We show that a hybrid (nuclear+quark matter) star can have a mass-radius relationship very similar to that predicted for a star made of purely nucleonic matter. We show this for a generic parameterization of the quark matter equation of state and also for an MIT bag model, each including a phenomenological correction based on gluonic corrections to the equation of state. We obtain hybrid stars as heavy as $2 M_{\odot}$ for reasonable values of the bag model parameters. For nuclear matter, we use the equation of state calculated by Akmal and coworkers using many-body techniques. Both mixed and homogeneous phases of nuclear and quark matter are considered.
\end{abstract}

Subject headings: dense matter — elementary particles — stars: neutron

Online material: color figures

\section{INTRODUCTION}

It has long been hypothesized that some compact stars might actually be "hybrid stars" containing cores of quark matter. The observationally accessible features of compact stars include their mass and radius, and there have been various investigations of how the presence of a quark matter core would affect the massradius relationship of a compact star. The general conclusion has been that quark matter softens the equation of state, so that hybrid stars are predicted to have lower maximum mass $\left(M \lesssim 1.7 M_{\odot}\right)$ than nuclear matter stars $\left(M \lesssim 2.2 M_{\odot}\right)$ (Lattimer \& Prakash 2001; Haensel 2003; Maieron et al. 2004; Alford \& Reddy 2003; Buballa et al. 2004; Banik \& Bandyopadhyay 2003; Burgio et al. 2002a, 2002b; Gocke et al. 2001; Schertler et al. 2000). (In this paper we only consider two-flavor nuclear matter. Introducing hyperons or kaon condensation into the nuclear matter also softens the equation of state and lowers the maximum mass [Lattimer \& Prakash 2001].) On the observational side, all mass measurements are currently compatible, at the $2 \sigma$ level, with $M \lesssim 1.7 M_{\odot}$ (Thorsett \& Chakrabarty 1999; Lattimer \& Prakash 2004). However, some are near the limit of compatibility, for example, pulsar J0751+1807 in a white dwarf-neutron star binary system, whose mass is currently measured at $2.1 \pm 0.4 M_{\odot}$ (Nice et al. 2005). The error bars on these measurements will decrease over time, so quark matter cores may seem to be on the point of being ruled out. Our purpose in this paper is to show that in fact it will be harder to rule out quark matter via $M(R)$ observations than these simple considerations indicate.

In an earlier paper (Alford \& Reddy 2003), we performed massradius calculations for hybrid and pure quark matter stars using a simple Massachusetts Institute of Technology (MIT) bag model equation of state. In that model, correlations arising due to quarkquark interactions were neglected. However, the effects of the strange quark mass and corrections to the equation of state due

\footnotetext{
${ }^{1}$ Affiliated with the Theoretical Division of Los Alamos National Laboratory for part of the work reported here.
}

to the pairing energy associated with color superconductivity (Rajagopal \& Wilczek 2002; Alford 2001; Rischke 2004; Schäfer 2003; Reddy 2002; Shovkovy 2004), the formation of quark Cooper pairs, were incorporated. We found that color superconductivity boosts the pressure of the quark matter relative to nuclear matter, lowering the transition density (at a fixed bag constant), but that the maximum mass was similar to that obtained in other work, namely, about $1.6 M_{\odot}$. We found that in order to form stars near this upper bound it was necessary to set the bag constant to a low value, so that quark matter is very nearly stable and the nuclear matter to quark matter $(\mathrm{NM} \rightarrow \mathrm{QM})$ phase transition occurs below nuclear saturation density, $n_{\mathrm{sat}}=0.16 \mathrm{fm}^{-3}$ : the heaviest stars consisted almost entirely of quark matter, with only a thin crust of nuclear matter.

\subsection{Overview of This Study}

In this paper we again use equations of state based on the MIT bag model for the quark matter, but we include an additional parameter that imitates the effect of including perturbative quantum chromodynamics (QCD) corrections.

For the first stage of this analysis ( $(3)$ we actually do not use any model at all. We write down a purely phenomenological quark matter equation of state, consisting simply of a power series expansion in the quark chemical potential, $\mu$,

$$
\Omega_{\mathrm{QM}}=-\frac{3}{4 \pi^{2}} a_{4} \mu^{4}+\frac{3}{4 \pi^{2}} a_{2} \mu^{2}+B_{\mathrm{eff}},
$$

where $a_{4}, a_{2}$, and $B_{\text {eff }}$ are independent of $\mu$. We show that for $a_{4} \approx 0.7$ (which we see below is physically reasonable), one can obtain heavy hybrid stars $\left(M \approx 2 M_{\odot}\right)$ while still ensuring that the $\mathrm{NM} \rightarrow$ QM phase transition occurs above nuclear saturation density. In fact, we show that it is possible to mimic the mass-radius behavior of nucleonic stars over a wide range of masses.

We then go on to the second stage of our analysis, in which we use a quark matter equation of state based on a physical model: 
competition between a "normal" unpaired quark matter phase and the color-flavor-locked (CFL) color-superconducting phase, in an MIT bag model formalism. This corresponds to giving $a_{2}$ and $B_{\text {eff }}$ a simple step-function dependence on the chemical potential, with the step occurring at the transition between these phases. The details are determined by the microscopic parameters of the model, the strange quark mass $m_{s}$ and the pairing gap $\Delta$.

The coefficient $a_{4} \equiv 1-c$ is a rough parameterization of QCD corrections to the pressure of the free-quark Fermi sea, and previous calculations (Fraga et al. 2001) show that the values $a_{4} \approx$ 0.7 and $c \approx 0.3$ are reasonable. The physical model also allows us to calculate the behavior of charged phases and hence to study inhomogeneous "mixed" phases of nuclear matter and quark matter.

The results from the quark matter model turn out to be very similar to those obtained with the simple parameterization. We also find that, given our current (and, likely, future) ignorance of the high-density values of basic parameters like the strange quark mass and bag constant, there are no characteristic features of the $M(R)$ relationship that could be used to verify the presence of color superconductivity in the quark matter core.

Our conclusion is that the maximum mass of hybrid stars is mainly determined by the size of the QCD corrections to the coefficient $a_{4}$ in the quark matter equation of state (1) and that for reasonable values of $a_{4}$ hybrid stars can be as heavy as $2 M_{\odot}$. This result is robust: it is not affected when we move up to a more sophisticated model or by the introduction of mixed phases. There is therefore little reason to expect it to change when the model is made even more complicated, e.g., by including kaoncondensed (Bedaque \& Schäfer 2002; Kaplan \& Reddy 2002; Kryjevski et al. 2005), crystalline (Alford et al. 2001a; Casalbuoni \& Nardulli 2004), mixed (Reddy \& Rupak 2005), or gapless (Shovkovy \& Huang 2003; Alford et al. 2004) phases or allowing continuous $\mu$-dependence in the strange quark mass or pairing gap (see below).

\subsection{Other Approaches}

Our calculations use a basic MIT bag model, in which the bag constant and quark masses are assumed to be density-independent. Other approaches are certainly possible. Within the MIT bag model one can use a density-dependent bag constant, although this does not appreciably change the maximum mass prediction (Burgio et al. 2002a, 2002b). The density-dependence of the constituent quark masses and the color superconducting gap $\Delta$ can be estimated by using a Nambu-Jona-Lasinio (NJL) model instead of a bag model, with coupled mean field Schwinger-Dyson equations for the masses and gaps. Such models give a high effective bag constant and quark masses and typically predict small numbers of strange quarks, with two-color-flavor superconductor (2SC) rather than CFL color superconductivity, but again the maximum masses turn out to be of order 1.6 $M_{\odot}$ (Buballa et al. 2004; Gocke et al. 2001), although folding certain Gaussian form factors into the four-fermion interaction can give masses up to $1.8 M_{\odot}$ (Grigorian et al. 2004). While these models are well motivated theoretically, their specific predictions relating to the density dependencies of quark masses and the effective bag constant remain untested. In this work we adopt a minimal approach and retain quartic and quadratic powers of the chemical potential in our expression for the free energy. This allows us to do a parameter study independent of any specific model. It would be interesting to see whether including a reasonable estimate of QCD corrections in the NJL model increases the maximum mass in that context also.

\section{THE NUCLEAR MATTER EQUATION OF STATE}

Our treatment of nuclear matter is completely standard: at densities above half the nuclear saturation density $\left(n_{\text {sat }}\right)$ we employ the equation of state of Akmal, Pandharipande, and Ravenhall (APR; Akmal et al. 1998). At lower densities we use the standard tabulated low-density equation of state (Baym et al. 1971; Negele \& Vautherin 1973). Our previous studies of hybrid stars (Alford \& Reddy 2003; Alford et al. 2001b) used the relativistic mean field Walecka model (Serot \& Walecka 1986) to describe nuclear matter or APR for $\beta$-stable charge-neutral nuclear matter. The relativistic mean field is an effective description of the nuclear matter that is constrained by properties of nuclear matter at saturation density. The mean field approximation ignores many-body correlations that could play an important role. APR use the variational chain summation (VCS) to include these correlations in calculating the equation of state of nucleon matter (Pandharipande \& Wiringa 1979; Akmal \& Pandharipande 1997). They employ a realistic nonrelativistic Hamiltonian with the Argonne $v_{18}$ (Wiringa et al. 1995) two-body potential and the Urbana IX (Pudliner et al. 1995) three-nucleon interaction. An equation of state as a function of baryon density and proton fraction, $x_{p}$, is obtained by interpolating between the pure neutron matter (PNM) $x_{p}=0$ and symmetric nuclear matter (SNM) $x_{p}=0.5$ results using a generalized Skyrme interaction containing momentum- and densitydependent delta function interactions (Pandharipande \& Ravenhall 1989) described below.

The energy is evaluated for a variational wave function that takes into account many-body correlation effects. It is composed as a symmetrized product of two-body correlation operators, $F_{i j}$, acting on the Fermi gas wave function. The correlation operators are written as a sum of terms that include operators appearing in the Hamiltonian. The two-body cluster contribution to the energy is minimized by $F_{i j}$ that satisfy the Euler-Lagrange equations determined within this Ansatz. Heuristically, two-body operators that appear in the Hamiltonian induce correlations between particles whose spatial dependence is approximated by solving a two-body Schrödinger-like equation subject to suitable boundary conditions. The variation of the wave function is effected at the twobody level by varying parameters appearing in this equation. Although this wave function neglects three-body correlations, it is estimated to be accurate to a few $\mathrm{MeV}$ nucleon ${ }^{-1}$ in SNM and about $1 \mathrm{MeV}$ nucleon ${ }^{-1}$ in PNM at nuclear density. This accuracy is achieved through the inclusion of many-body effects via the VCS technique. Comparable accuracy is obtained with the other many-body techniques like the Brueckner-Bethe-Goldstone method of Baldo et al. (1997).

The APR equation of state exhibits a transition from a lowdensity phase (LDP) to a high-density phase (HDP) having spinisospin order, possibly due to neutral pion condensation, in PNM at a density of $\sim 0.20 \mathrm{fm}^{-3}$ and in SNM at $\sim 0.32 \mathrm{fm}^{-3}$. The VCS calculations of the energy of PNM and SNM are extrapolated to general values of $x_{p}$ using a function of the form

$$
\begin{aligned}
\epsilon_{N}\left(\rho, x_{p}\right)= & {\left[\frac{\hbar^{2}}{2 m_{N}}+f\left(\rho, x_{p}\right)\right] \tau_{p}+\left[\frac{\hbar^{2}}{2 m_{N}}+f\left(\rho, 1-x_{p}\right)\right] \tau_{n} } \\
& +g\left(\rho, x_{p}=0.5\right)\left[1-\left(1-2 x_{p}\right)^{2}\right] \\
& +g\left(\rho, x_{p}=0\right)\left(1-2 x_{p}\right)^{2}
\end{aligned}
$$

motivated by a generalized Skyrme interaction. Here $\epsilon_{N}$ is the total nuclear energy density, $\tau_{n, p}$ are the neutron and proton Fermi gas kinetic densities, $f\left(\rho, x_{p}\right)$ and $f\left(\rho, 1-x_{p}\right)$ are functions that parameterize the effective mass of the nucleons, and 
$g\left(\rho, x_{p}=0\right)$ and $g\left(\rho, x_{p}=0.5\right)$ are potential energy terms. These functions are parameterized to fit the energies of PNM and SNM calculated in VCS. Separate parameterizations are used in the LDP and HDP for the functions $g$. The energy density of nuclear matter is used to determine the allowed equation of state of hybrid stars for the case of a sharp transition to quark matter and to determine the allowed phases for a mixed transition to quark matter.

\section{A SIMPLE PHENOMENOLOGICAL QUARK MATTER EQUATION OF STATE}

One of the main points of this paper is to show the effects of including a parameter in the quark matter equation of state that roughly incorporates the effects of gluon-mediated QCD interactions between the quarks in the Fermi sea. We first do this in the context of a simple parameterization of the quark matter equation of state and show that the resulting hybrid stars can have mass-radius relations very similar to those of pure nuclear stars, with masses up to $2 M_{\odot}$. Below $(\S 4)$ we show that these conclusions remain true in a more sophisticated model.

The simple phenomenological parameterization of the quark matter equation of state is

$$
\begin{gathered}
\Omega_{\mathrm{QM}}=-\frac{3}{4 \pi^{2}} a_{4} \mu^{4}+\frac{3}{4 \pi^{2}} a_{2} \mu^{2}+B_{\mathrm{eff}}, \\
a_{4} \equiv 1-c
\end{gathered}
$$

where the parameters $a_{4}, a_{2}$, and $B_{\text {eff }}$ are independent of $\mu$.

The quartic coefficient $a_{4}=1-c$.-For quark matter consisting of three flavors of free noninteracting quarks, $c=0$, so $a_{4}=1$ (see the discussion of the physical model of quark matter in $\S 4$ ). However, once QCD corrections are taken into account, we expect $c \neq 0$.

The QCD corrections to the quark matter equation of state were first evaluated to $O\left(\alpha_{s}^{2}\right)$ by Freedman and McLerran (Farhi \& Jaffe 1984; Freedman \& McLerran 1977a, 1977b, 1977c, 1978; Baluni 1978); then Fraga et al. (2001, hereafter FPS) showed that the $O\left(\alpha_{s}^{2}\right)$ pressure for three massless flavors can be approximated by a bag model-inspired form,

$$
P_{\alpha_{s}^{2}} \approx \frac{3}{4 \pi^{2}}(1-c) \mu^{4}-B_{\mathrm{eff}} .
$$

Matching to the $O\left(\alpha_{s}^{2}\right)$ perturbative calculations in the interval $\mu \simeq 300-600 \mathrm{MeV}$, they found that $B_{\text {eff }}$ varies widely with renormalization scale (see also Andersen \& Strickland 2002), but they consistently find $c \approx 0.37$. We do not use FPS's specific values of $B_{\text {eff }}$ and $c$ because, as they observe, the QCD coupling is strong at the density of interest for compact star physics, so there is no reason to expect the leading order calculation to be accurate. However, we take their results as indicating that QCD corrections are not negligible, so one should include $c$ as an additional parameter in the quark matter equation of state, with a value of order 0.3 .

The quadratic coefficient $a_{2}$.-As we see in $\S 4$, the $\mu^{2}$ term can arise from the strange quark mass (which increases the free energy) or color superconductivity (which reduces it). If chiral symmetry remains broken in the light quark sector due to a robust $\langle\bar{q} q\rangle$ condensate, then the large $(\sim 100-300 \mathrm{MeV})$ constituent quark masses of the up and down quarks would also result in a $\mu^{2}$ term similar to that due to the strange quark mass. For CFL quark matter, $a_{2}=m_{s}^{2}-4 \Delta^{2}$. For now, we simply include $a_{2}$ as a phenomenological parameter.
The bag constant and the transition density $\rho_{c}$.- -In our parameterization the effective bag constant simply accounts for the free energy contribution that is independent of $\mu$. While this is related to the vacuum pressure, its numerical value in our parameterization need not be the same as in early bag model studies of hadron phenomenology (Chodos et al. 1974). The effective bag constant is unknown and difficult to calculate or measure, so we do not use it as a parameter, since that would obscure the fact that part of the effect of varying other parameters may simply be a renormalization of the unknown parameter $B_{\text {eff. }}$ To expose the physically significant effects of varying $a_{4}, a_{2}$, and $B_{\text {eff }}$, we specify a more physical quantity, the maximum density $\rho_{c}$ of nuclear matter, i.e., the density at which the NM $\rightarrow$ QM transition occurs. The structure of the star is then calculated as a function of $a_{4}, a_{2}$, and $\rho_{c}$.

Two subtle points arise in such a reparameterization. First, as we see below, for values of the perturbative correction parameter $c$ around 0.3 , the quark matter and APR nuclear matter equations of state have almost exactly the same shape over a wide range of pressures. This can lead to multiple phase transitions back and forth between NM and QM. Of course, when the two phases have almost identical equations of state, it does not matter (for mass and radius calculations) where transitions between them occur. We therefore simply choose $\rho_{c}$ to be associated with the location of the first transition.

Second, when we use the more complicated model in $\S 4$, we allow for the possibility of mixed phases, which blur out the NM $\rightarrow$ QM transition over a range of densities and pressures, making it hard to identify "the" transition density. However, for the purpose of fixing the bag constant, we do not have to allow mixed phases. We therefore define $B_{\text {eff }}\left(a_{4}, a_{2}, \rho_{c}\right)$ as the value of the bag constant that would give a sharp NM $\rightarrow$ QM transition at a nuclear matter density $\rho_{c}$ if only charge neutral bulk phases were permitted (as would happen if the NM-QM surface tension were $\left.\geq 40 \mathrm{MeV} \mathrm{fm}^{-3}\right)$.

\subsection{The Physical Effects of the "Perturbative Correction" Parameter}

We now discuss the physical importance of the QCD correction $c$. Firstly, it is clear analytically that, at a fixed bag constant, $c$ has very little effect on the relation $E(p)$ between energy density and pressure for quark matter, which enters into the Tolman-Oppenheimer-Volkoff (TOV) equation (Tolman 1939; Oppenheimer \& Volkoff 1939). This can be seen by setting $a_{2}=$ 0 (i.e., neglecting quark masses and pairing), in which case

$$
\begin{aligned}
p & =(1-c) \frac{3 \mu^{4}}{4 \pi^{2}}-B_{\mathrm{eff}}, \\
E & =3(1-c) \frac{3 \mu^{4}}{4 \pi^{2}}+B_{\mathrm{eff}}, \\
\Rightarrow E & =3 p+4 B_{\mathrm{eff}} \quad \text { independent of } c .
\end{aligned}
$$

However, this does not mean that $c$ is unimportant. Clearly $c$ makes a dramatic difference to $p(\mu)$, so it strongly affects the position of the $\mathrm{NM} \rightarrow \mathrm{QM}$ transition. Moreover, as described above, we are not working at fixed $B_{\text {eff }}$ : when we change $c$, we keep the transition density $\rho_{c}$ fixed, with a resulting change in $B_{\text {eff. }}$.

We illustrate the effect of nonzero $c$ in Figure 1, which shows $p(\mu)$ and $E(\mu)$ for APR nuclear matter and for the phenomenological description of quark matter, with $a_{2}=(150 \mathrm{MeV})^{2}$, for the cases $c=0$ and 0.3 (tuning $B_{\text {eff }}$ to keep the transition density $\rho=1.5 n_{\text {sat }}$ ). We see that the $c=0.3$ quark matter equation of state is very similar to APR over the pressure range $10-200 \mathrm{MeV} \mathrm{fm}^{-3}$. 

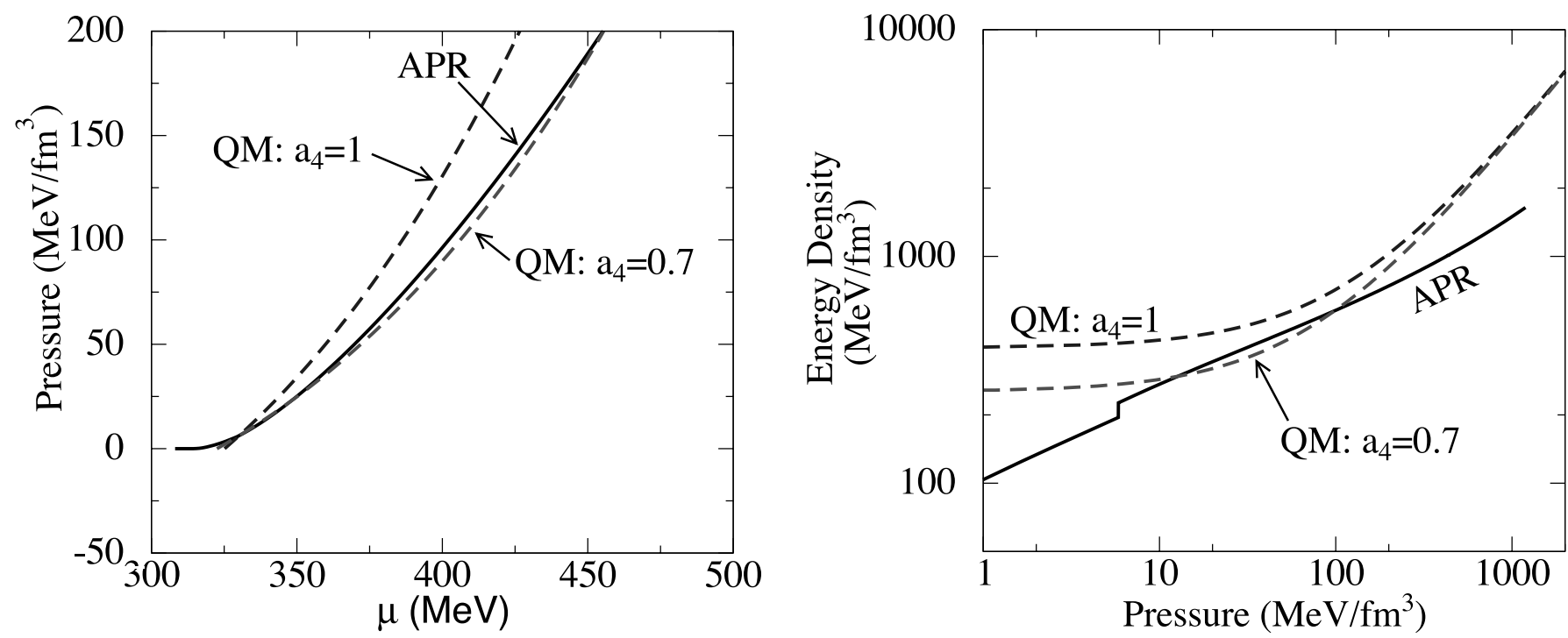

FIG. 1.-Equations of state for APR nuclear matter (solid curve) and for quark matter with the phenomenologically parameterized equation of state (3) with $a_{2}=$ $(150 \mathrm{MeV})^{2}$ [dashed curves: upper curve has no perturbative correction $(c=0)$, lower one has $c=0.3$ ]. For each quark matter equation of state, the bag constant $B_{\text {eff }}$ was fixed by requiring that nuclear matter give way to quark matter at $\rho_{c}=1.5 n_{\text {sat }}$. The figure shows that when we include perturbative-type corrections, quark matter has a $p(\mu)$ relation almost identical to that of APR nuclear matter, and its $E(p)$ is also very similar over the range of pressures that is relevant to compact star masses. [See the electronic edition of the Journal for a color version of this figure.]

In fact, close examination of the $p(\mu)$ relationship shows that there are three phase transitions with increasing density: from APR nuclear matter at low density to quark matter, then back to APR, then back to quark matter again. On this basis, we expect stars containing quark matter with $c \approx 0.3$ to show $M(R)$ curves very similar to those of nuclear matter stars, making them correspondingly difficult to rule out from $M(R)$ measurements alone. In $\S 3.3$ we see that this is indeed the case.

The $p(\mu)$ relations for $c=0.3$ quark matter and APR are so similar that it can be difficult to say where the phase transition really occurs. In Figure 2 we show the pressure difference for the parameters of Figure $1\left(B_{\mathrm{eff}}^{1 / 4}=141.2 \mathrm{MeV}\right.$, solid curve $)$ and for a higher value of the bag constant $\left(B_{\text {eff }}^{1 / 4}=141.5 \mathrm{MeV}\right.$, dashed curve). At $B_{\text {eff }}^{1 / 4}=141.2 \mathrm{MeV}$, the three transitions are clearly visible. We see that when the bag constant is raised to $B_{\text {eff }}^{1 / 4}=$ 141.5 MeV to obtain a transition at higher density, the value of the chemical potential at the transition, and hence the density, jumps discontinuously from $\mu=338 \mathrm{MeV}\left(\rho=1.9 n_{\text {sat }}\right)$ to $\mu=$ $458 \mathrm{MeV}\left(\rho=4.3 n_{\mathrm{sat}}\right)$. This is illustrated in Figure 2 (right). It is therefore technically impossible to choose $B_{\text {eff }}$ so as to obtain a (first) transition density in the range $1.9 n_{\text {sat }}<\rho_{c}<4.3 n_{\text {sat }}$.

It is very interesting to speculate on the possibility of multiple phase transitions inside compact stars, but this feature is highly sensitive to the precise relative shapes of our quark matter and APR equations of state. We cannot claim to know these to the level of accuracy (a few percent, i.e., a few million electron volts per cubic fermi) that would be required to say whether the number of transitions is one, three, or even five. Our main
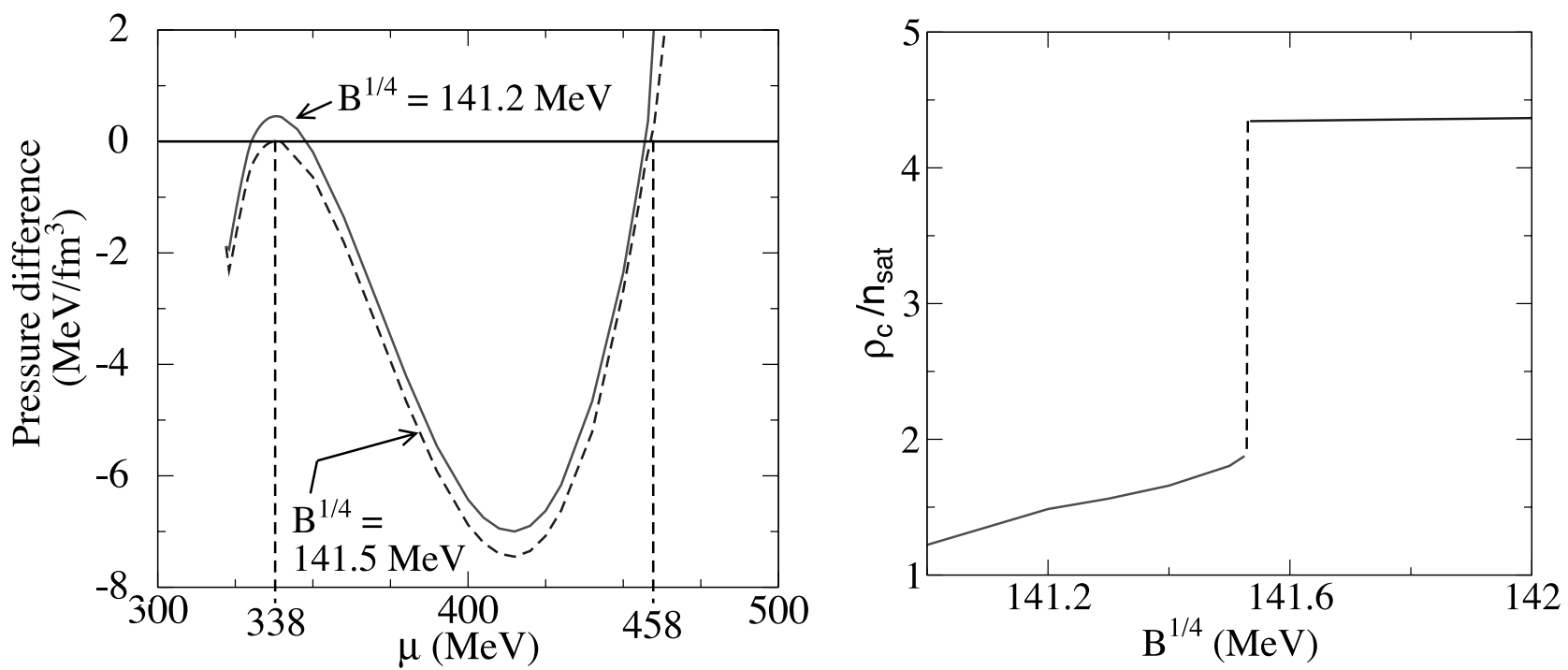

FIG. 2.-Pressure difference between quark matter and APR nuclear matter for $a_{4}=0.7, a_{2}=(150 \mathrm{MeV})^{2}$, illustrating how the near-identity of their $p(\mu)$ relations leads to difficulties in defining a unique transition density. In the left panel, the solid curve is for $B_{\text {eff }}^{1 / 4}=141.2 \mathrm{MeV}$ and shows three transitions, the first occurring at $\mu=324.4 \mathrm{MeV}$, corresponding to $\rho_{c}=1.5 n_{\text {sat }}$ (Fig. 1). Raising $B_{\text {eff }}$ gives a transition at higher $\mu$, but it is technically impossible to achieve a (first) transition in the range $338 \mathrm{MeV}<\mu<458 \mathrm{MeV}\left(1.9 n_{\text {sat }}<\rho<4.3 n_{\text {sat }}\right)$ at $B_{\text {eff }}^{1 / 4}=141.5 \mathrm{MeV}$ (dashed curve) the transition jumps from the bottom of this range to the top. The right panel illustrates how this translates to a discontinuous behavior in dependence of $\rho_{c}$ on $B_{\mathrm{eff}}$. [See the electronic edition of the Journal for a color version of this figure.] 
message in this paper is that for reasonable values of the quark matter parameters, the equations of state may be very similar. A realistic interpretation of Figure 2 is that at $B_{\text {eff }}^{1 / 4}=141.5 \mathrm{MeV}$ the critical density jumps rapidly from $1.9 n_{\text {sat }}$ to $4.3 n_{\text {sat }}$. Any $\rho_{c}$ in that range therefore corresponds to $B_{\text {eff }}^{1 / 4}=141.5 \mathrm{MeV}$. (Pictorially, this corresponds to blurring out the curves in Figure 2 by a few million electron volts per cubic fermi.) This is the procedure we follow in determining $B_{\text {eff }}$ for given $\rho_{c}, m_{s}$, and $\Delta$ when we investigate how the maximum compact star mass depends on these parameters.

One can extrapolate from Figures 1 and 2 to predict what will happen for even larger values of $c$. For suitable values of the bag constant, there will be a small range of densities (near nuclear density) in which quark matter has higher pressure than APR nuclear matter; then at intermediate densities APR will be favored again; and finally quark matter will win at high densities. When such equations of state are used to construct compact stars, the result will be a star containing a shell of quark matter with APR nuclear matter outside and inside it. This also leads to large masses, but only because most of the star is nuclear matter. In the rest of this paper we discuss values of $c$ up to 0.3 , which is suggested by Fraga et al.'s fit to the two-loop equation of state (Fraga et al. 2001) and also offers the possibility of heavy stars with a considerable quark matter fraction.

\subsection{Mass-Radius Relationship: A Hybrid Star That "Looks Nuclear"}

Before surveying a wide range of values of the parameters $a_{4}=1-c, a_{2}$, and $\rho_{c}$, we first display the $M(R)$ curve for some specific cases, showing how masses approaching $2 M_{\odot}$ can be achieved. The $M(R)$ relation is obtained by solving the TOV equation in the standard way, as described by Alford \& Reddy (2003). We choose a range of central pressures and integrate the TOV equation outward until the pressure drops to zero, which marks the surface of the star, and the integrated energy density yields the mass (Glendenning 1997). In Figure 3 we show the result-

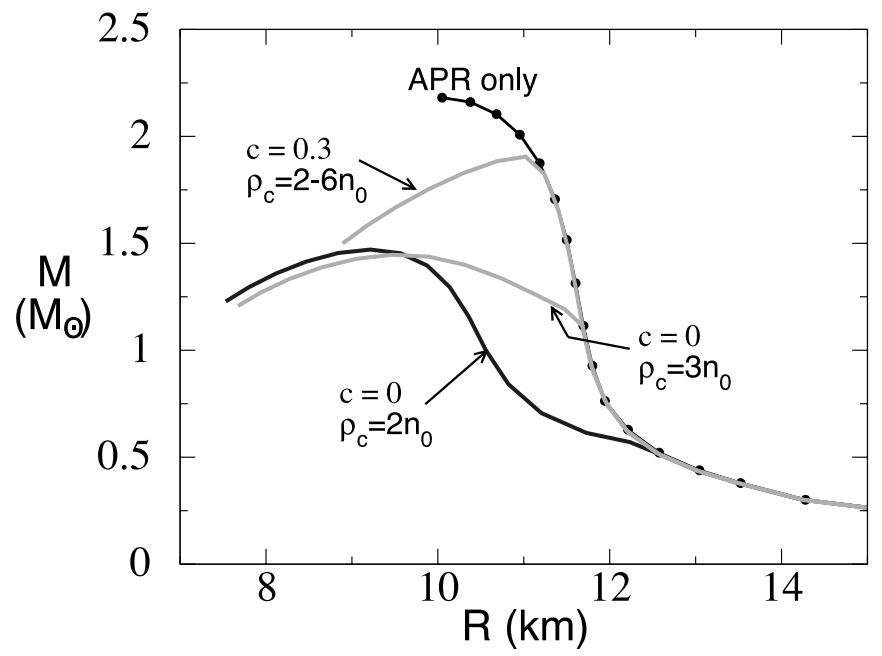

FIG. 3.-The $M(R)$ relationship for hybrid stars involving quark matter obeying the phenomenological equation of state (3) with $a_{2}=(180 \mathrm{MeV})^{2}$. In each case the bag constant was chosen to give the desired transition density. The line with filled circles is the $M(R)$ relation for a pure nuclear APR star. All the phases are neutral and homogeneous. We see that the maximum mass $M_{\max }$ is very sensitive to the QCD correction $c$ but not to the transition density. For $c=0.3$, which is close to the value suggested by Fraga et al. (2001), $M_{\max } \approx$ $1.9 M_{\odot}$. Note also that the $c=0.3$ equation of state is so similar to APR that its $M(R)$ curve is almost identical to that of pure APR. [See the electronic edition of the Journal for a color version of this figure.]

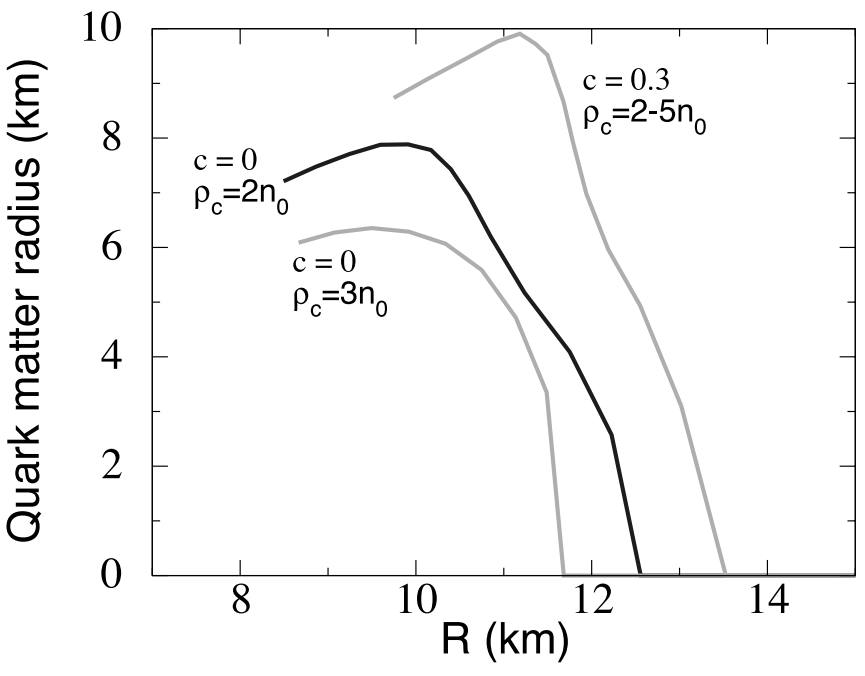

FIG. 4.-Radius at which the first (lowest density) transition from nuclear matter to quark matter occurs, for the families of stars whose $M(R)$ relations were given in Fig. 3. Note that this radius is quite large for the heavier stars. In the $c=0.3$ case there may be transitions back and forth between quark and nuclear matter at higher density (smaller radii). [See the electronic edition of the Journal for a color version of this figure.]

ing $M(R)$ curves for quark matter with $a_{2}=(180 \mathrm{MeV})^{2}$. We performed calculations with $c=0$ and 0.3 (Fraga et al. 2001). We tuned the bag constant to give a range of homogeneous neutralmatter transition densities $\rho_{c}=(2-4) n_{\text {sat }}$.

The most obvious feature is the dependence on $c$ : compared to the stars with $c=0$, hybrid stars containing quark matter with a larger perturbative correction $c=0.3$ are significantly heavier and a little larger, with masses approaching $2 M_{\odot}$ and radii around $11 \mathrm{~km}$. It is also striking that stars with $c=0.3$ have an $M(R)$ relation essentially identical to that of APR nuclear matter, up to masses around $1.9 M_{\odot}$. This is because quark matter with $c=0.3$ has an equation of state very close to that of APR nuclear matter over the relevant range of pressures. This was discussed in $\S 3.1$.

In Figure 4 we show, for the same family of stars, the radius at which the first (lowest density) transition from nuclear matter to quark matter occurs. Note that this radius is quite large for the heavier stars. In the $c=0.3$ case there may be transitions back and forth between quark and nuclear matter as one goes deeper into the star, because the equations of state are so similar. While such multiple transitions cannot be ruled out a priori, we suspect that they are not physical. They are an artifact of our model description of the nuclear and quark phases and not a robust prediction. Here and in the rest of the paper we ignore this possibility and entertain only one transition from nuclear to quark matter, beyond which we use the quark matter equation of state. While this will make little difference to the structure of the star, since the nuclear and quark matter equations of state are both very similar in this regime, it would have important consequences for transport properties.

\subsection{Maximum Mass as a Function of Quark Matter Parameters}

Having seen that with a perturbative correction $c$ set to a reasonable value we can increase the maximum hybrid star mass, we now look at how maximum hybrid star mass depends on the parameters of our phenomenological equation of state (3): $a_{4}, a_{2}$, and the transition density $\rho_{c}$.

In Figure 5 we plot the maximum mass, $M_{\max }$, obtained by varying the central pressure and choosing the heaviest resulting 


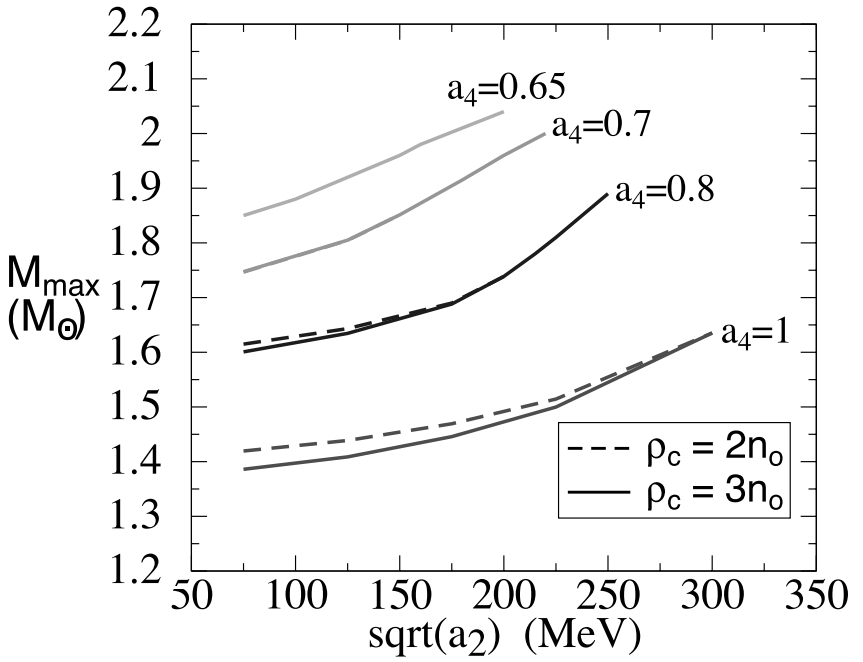

FIG. 5.-Dependence of $M_{\max }$ on $a_{2}$ for hybrid stars involving quark matter obeying the phenomenological equation of state (3). Increasing $a_{2}$ decreases $B_{\text {eff }}$ at fixed $\rho_{c}$, giving rise to larger maximum masses. We show results for two transition densities, $\rho_{c}=3 n_{\text {sat }}$ and $2 n_{\text {sat }}$. The maximum mass is not very sensitive to the transition density. [See the electronic edition of the Journal for a color version of this figure.]

stable star that contains some quark matter. We calculated $M_{\max }$ as a function of $a_{2}$, for $a_{4}=1,0.8,0.7$, and 0.65 . In each case we repeated the calculation for two different transition densities, $\rho_{c}=3 n_{\text {sat }}$ (solid lines) and $\rho_{c}=2 n_{\text {sat }}$ (dashed lines). The lines in Figure 5 end when there is no longer a stable hybrid star. This corresponds to the hybrid star branch in the $M(R)$ plot having a positive slope along its whole length.

We see that masses up to $2 M_{\odot}$ can be obtained by choosing a small value of $a_{4}$ and setting $a_{2}^{1 / 2}$ to an appropriate value in the range $150-250 \mathrm{MeV}$. (Recall that in unpaired quark matter $a_{2}^{1 / 2}$ corresponds to the strange quark mass.) This is understandable, since the effective bag constant must decrease with increasing $a_{2}$ at fixed $\rho_{c}$ : it is well known that in stars dominated by quark matter, $M_{\text {max }} \sim B_{\text {eff }}^{-1 / 2}$ (Witten 1984).

One might want to ask how large the quark matter core is for the heaviest stars, but this question does not have an easy answer. For $a_{4} \sim 0.7(c \sim 0.3)$, the quark matter and nuclear matter equations of state are so similar that the transition density is not precisely defined (as we saw in Fig. 2), so it is not clear where the transition occurs: there is a whole range of transition densities that correspond to the same bag constant. This is why, for lower values of $a_{4}$, the maximum mass is not very sensitive to the transition density. Figure 4 shows that the first transition from nuclear to quark matter occurs at quite large radii for the heavy stars.

\subsection{Conclusion}

We have seen that the very simple phenomenological parameterization (3) of the quark matter equation of state allows us to survey the main effects of the various parameters and makes it clear that $a_{4}=1-c$ is the most important in determining the maximum mass. With a value corresponding to free-quark matter $(c=0)$, we find a maximum hybrid star mass of about $1.6 M_{\odot}$, similar to the value found in previous studies. With a value corresponding to QCD corrections of a plausible strength $(c \approx$ 0.35 ), we find a maximum mass of about $2 M_{\odot}$.

In $\S 4$ we show that this conclusion is robust. We repeat our analysis with a more complicated quark matter equation of state, based on the expected physics of dense quark matter. We allow competition between paired and unpaired phases and mixed quark and nuclear matter phases. We find that these factors have no effect on our essential findings regarding the maximum mass of hybrid stars.

\section{A MORE SOPHISTICATED APPROACH: A PHYSICAL MODEL OF QUARK MATTER AND MIXED PHASES}

The model we use in this section is more complicated and physically well founded than the phenomenological parameterization of $\S 3$, but it is still a relatively simple MIT bag model. We allow competition between two phases of quark matter, the three-flavor unpaired phase and the CFL color-superconducting phase. The phase with the lowest free energy (highest pressure) is favored.

The parameters of this model are the strange quark mass $m_{s}$, the CFL pairing gap $\Delta$, the density $\rho_{c}$ of the nuclear-to-quarkmatter transition (which determines the bag constant), and a QCD-inspired correction parameter $c$. This is similar to the phenomenological parameterization of $\S 3$, but the effective parameter $a_{2}$ is now calculated within a model.

At asymptotically high chemical potential on the order of $10^{8} \mathrm{MeV}$, the CFL phase is known to be the ground state of three-flavor quark matter, although it may persist to much lower chemical potentials. At lower densities the strange quark mass becomes nonnegligible relative to the chemical potential, and at $m_{s}^{2} / \mu=4 \Delta$ there is a transition to unpaired quark matter (Alford $\&$ Rajagopal 2002). ${ }^{2}$ When we include the nuclear matter, there is a three-way competition between nuclear matter, unpaired quark matter, and CFL quark matter.

Now that we have a physical model in hand, we can calculate the pressure of charged, as well as neutral, phases of quark matter. This means that we can study mixed, as well as homogeneous, phases. The transition from nuclear matter to quark matter can proceed via a mixed phase (Glendenning 1992), in which there is charge separation, and a positively charged nuclear phase interpenetrates with a negatively charged quark matter phase, yielding a globally neutral inhomogeneous phase. This will only occur if the surface tension at the boundary of the two phases is low enough. Otherwise there will be a sharp interface between the two homogeneous neutral phases. The critical surface tension is $\sigma_{c} \approx 40 \mathrm{MeV} \mathrm{fm}^{-3}$ (Heiselberg et al. 1993; Alford et al. 2001b). Since the surface tension is completely unknown, we separately consider both mixed phases and sharp interfaces in our calculations.

\subsection{Unpaired Three-Flavor Quark Matter}

In the naive bag model, in which perturbative corrections are ignored, the free energy of free-quark matter consists of the kinetic contribution from a degenerate free gas of three colors of relativistic quarks and the negative vacuum pressure from the bag constant, $B_{\text {eff }}$,

$$
\begin{aligned}
& \Omega_{\mathrm{unp}}\left(\mu_{u}, \mu_{d}, \mu_{s}\right)= \\
& \frac{3}{\pi^{2}} \sum_{i=u, d, s} \int_{0}^{\sqrt{\mu_{i}^{2}-m_{i}^{2}}} d p p^{2}\left(\sqrt{p^{2}+m_{i}^{2}}-\mu_{i}\right)+B_{\mathrm{eff}},
\end{aligned}
$$

\footnotetext{
2 Actually, before that, at $m_{s}^{2}=2 \mu \Delta$, there is a transition to a gapless phase (Alford et al. 2004, 2005), and at $m_{s} \sim m_{\text {light }}^{1 / 3} \Delta^{2 / 3}$ there is the possibility of $K^{0}$ condensation in the CFL phase (Bedaque \& Schäfer 2002; Kaplan \& Reddy 2002; Kryjevski et al. 2005): we ignore these additional complications because their contributions to the pressure are of order $m_{s}^{4}$, which is just a renormalization of the bag constant, which for us is a nonphysical parameter.
} 
where $\mu_{u}=\mu-(2 / 3) \mu_{e}, \mu_{d}=\mu_{s}=\mu+(1 / 3) \mu_{e}$ are the individual quark chemical potentials and $\mu$ and $\mu_{e}$ are the baryon and electron chemical potentials, respectively. We may neglect the quark masses of the up and down quarks in quark matter, since $m_{\text {light }} \sim m_{u} \sim m_{d} \ll \mu$. On the other hand, the strange quark mass is not negligible compared to $\mu$. In this work we assume that $m_{s}<\mu$ so that an expansion in powers of $m_{s} / \mu$ is meaningful and study three-flavor quark matter.

In neutral unpaired quark matter, the electron chemical potential is determined by the condition of charge neutrality and is given by

$$
\mu_{e}=\frac{m_{s}^{2}}{4 \mu}-\frac{m_{s}^{4}}{48 \mu^{3}}+O\left(\frac{m_{s}^{6}}{\mu^{5}}\right) .
$$

Substituting this in equation (6) and expanding in powers of $m_{s} / \mu$, we obtain

$$
\begin{aligned}
\Omega_{\text {unpaired }}^{\text {neutral }}(\mu)= & -\frac{3}{4 \pi^{2}}(1-c) \mu^{4} \\
& +\frac{3 \mu^{2} m_{s}^{2}}{4 \pi^{2}}+\left[12 \log \left(\frac{m_{s}}{2 \mu}\right)-7\right] \frac{m_{s}^{4}}{32 \pi^{2}} \\
& +\frac{5 m_{s}^{6}}{576 \pi^{2} \mu^{2}}+B_{\text {eff }}+O\left(\frac{m_{s}^{8}}{\mu^{4}}\right),
\end{aligned}
$$

where we have dropped terms of order $m_{s}^{8} / \mu^{4}$ and higher and introduced a parameter $c$ corresponding to the QCD-inspired corrections of Fraga et al. (2001), just as we did in $\S 3$. From equation (8) we see that the expansion in powers of $m_{s} / \mu$ is rapidly convergent, even for $m_{s} \sim \mu$. The contribution to the free energy from the $m_{s}^{6} / \mu^{2}$ term is less than one part in $10^{4}$ of the $\mu^{4}$ term when $m_{s} \sim 300 \mathrm{MeV}$ and $\mu=350 \mathrm{MeV}$. This means that neutral unpaired quark matter is really just a particular case of the phenomenological parameterization explored in $\S 3$.

The FPS parameterization relies on the analysis of Freedman and McLerran, which is rigorous for massless quarks (Farhi \& Jaffe 1984; Freedman \& McLerran 1977a, 1977b, 1977c, 1978; Baluni 1978). We therefore apply the effects of the "perturbative" QCD correction only to the $\mu^{4}$ (i.e., massless) part of the free energy. Again, this is similar to the phenomenological parameterization, where the QCD correction is a modification of the coefficient of the $\mu^{4}$ term.

If we do not impose the neutrality condition but expand in powers of $\mu_{e} / \mu$ as well as $m_{s} / \mu$, we find that the expansion does not converge nearly as well, so for charged unpaired quark matter we must use the full form of the free energy equation (6).

\subsection{Color-Flavor-locked Quark Matter}

In CFL matter, the pairing locks the Fermi momenta of all the quarks to a single value, requiring the number densities of up, down, and strange quarks to be equal (Rajagopal \& Wilczek 2001; Steiner et al. 2002). This costs free energy, which is offset by the pairing contribution

$$
\Omega_{\Delta}=-\frac{3}{\pi^{2}} \Delta^{2} \mu^{2}+O\left(\Delta^{4}\right) .
$$

Calculations of $\Delta$ with effective interactions yield values in the range 10-100 MeV (Rajagopal \& Wilczek 2001; Alford 2001; Rischke 2004; Schäfer 2003; Reddy 2002; Shovkovy 2004) for $\mu$ in the range $300-600 \mathrm{MeV}$. So it is reasonable to retain only the leading order (in powers of $\Delta$ ) contribution. As in the case of the strange quark mass, the $\Delta^{4}$ contribution has a weak (log- arithmic) dependence on $\mu$, and its contribution to the equation of state is indistinguishable from $B_{\text {eff. }}$.

When color and electric neutrality is imposed, there are no electrons in the CFL phase, since there are equal numbers of up, down, and strange quarks, so the electron chemical potential $\mu_{e}=$ 0 . Expanding the free energy in powers of $m_{s} / \mu$, we find (Alford et al. 2001b)

$$
\begin{aligned}
\Omega_{\mathrm{CFL} \text { quarks }}^{\text {neutral }}= & -\frac{3}{4 \pi^{2}}(1-c) \mu^{4}+\frac{3 m_{s}^{2} \mu^{2}}{4 \pi^{2}}-\frac{3 \Delta^{2} \mu^{2}}{\pi^{2}} \\
& +\left[12 \log \left(\frac{m_{s}}{2 \mu}\right)-1\right] \frac{m_{s}^{4}}{32 \pi^{2}}+B_{\text {eff }}+O\left(\frac{m_{s}^{6}}{\mu^{2}}\right) \\
= & \Omega_{\text {unpaired }}^{\text {neutral }}+\frac{3 m_{s}^{4}-48 \Delta^{2} \mu^{2}}{16 \pi^{2}}+O\left(\frac{m_{s}^{6}}{\mu^{2}}\right),
\end{aligned}
$$

where we have assumed that the unpaired and CFL phases have the same bag constant.

We see from the first line of this expression that the equation of state of neutral CFL matter is controlled by the strange quark mass and pairing gap in a combination that corresponds to the parameter $a_{2}$ in the phenomenological parameterization (3), if we identify

$$
a_{2}=m_{s}^{2}-4 \Delta^{2} .
$$

Note that the $m_{s}^{4}$ terms do not follow such an identification, but they are irrelevant because they are $\mu$-independent, so they are a renormalization of the bag constant, which we choose to give some specified transition density. Therefore, the neutral CFL equation of state, to the order that we have expanded it, is rigorously a function of the QCD-inspired correction $c$, the chosen transition density $\rho_{c}$, and $m_{s}^{2}-4 \Delta^{2}$. However, the equation of state for neutral quark matter in general is not rigorously a function of $m_{s}^{2}-4 \Delta^{2}$ only, because for a given $m_{s}$ and $\Delta$ there is competition between the neutral CFL phase and the neutral unpaired phase, whose equation of state is affected by $m_{s}$ alone. However, we expect the dependence on the linearly independent variable $4 m_{s}^{2}+\Delta^{2}$ to be very weak. By independently varying $m_{s}$ and $\Delta$, we have verified that this is the case. Of course, mixed phases are complicated by the fact that they involve charged quark matter whose equation of state depends on $\mu_{e}$ as an additional parameter.

We want to construct mixed phases, so we must also know the equation of state for charged CFL matter with $\mu_{e} \neq 0$. This has contributions from the quarks, the electrons, and from the Goldstone bosons. The Goldstone bosons arise due to the spontaneous breaking of chiral symmetry, analogous to the mechanism in vacuum (Alford et al. 1999). The quark contribution is independent of $\mu_{e}$ : CFL-paired quarks form an insulator with gap $\Delta$. So as long as $\mu_{e}<\Delta$ there are no charged quasi-quark excitations, so $Q=\partial \Omega / \partial \mu_{e}=0$.

The Goldstone bosons are a consequence of spontaneous breaking of chiral symmetry (Alford et al. 1999, 2001b) and, like the octet of pseudoscalar mesons of QCD in vacuum, can be described by an effective chiral field theory (Son \& Stephanov 2000). When the electron chemical potential exceeds the mass of the lightest negatively charged meson, which in the CFL phase is the $\pi^{-}$, these mesons condense (Bedaque \& Schäfer 2002; Kaplan \& Reddy 2002; Kryjevski et al. 2005). The free energy contribution of the meson condensate is

$$
\Omega_{\mathrm{CFL}}^{\mathrm{GB}}\left(\mu, \mu_{e}\right)=-\frac{1}{2} f_{\pi}^{2} \mu_{e}^{2}\left(1-\frac{m_{\pi}^{2}}{\mu_{e}^{2}}\right)^{2},
$$


where the parameters are (Son \& Stephanov 2000)

$$
f_{\pi}^{2}=\frac{(21-8 \ln 2) \mu^{2}}{36 \pi^{2}}, \quad m_{\pi^{-}}^{2}=\frac{3 \Delta^{2}}{\pi^{2} f_{\pi}^{2}} m_{s}\left(m_{u}+m_{d}\right) .
$$

We used $m_{u}=3.75 \mathrm{MeV}$ and $m_{d}=7.5 \mathrm{MeV}$. Finally, the free energy contribution from electrons and muons is given by

$$
\Omega^{\text {leptons }}\left(\mu_{e}\right)=\sum_{i=e^{-}, \mu^{-}} \frac{1}{\pi^{2}} \int_{0}^{\sqrt{\mu_{e}^{2}-m_{i}^{2}}} d p p^{2}\left(\sqrt{p^{2}+m_{i}^{2}}-\mu_{e}\right) .
$$

The total free energy for CFL quark matter is then

$$
\Omega_{\mathrm{CFL}}^{\text {charged }}\left(\mu, \mu_{e}\right)=\Omega_{\mathrm{CFL} \text { quarks }}^{\text {neutral }}(\mu)+\Omega_{\mathrm{CFL}}^{\mathrm{GB}}\left(\mu, \mu_{e}\right)+\Omega^{\text {leptons }}\left(\mu_{e}\right) \text {. }
$$

\subsection{A Bag Model Hybrid Star that Masquerades as a Neutron Star}

Using the bag model equations of state for unpaired and CFL quark matter, we can proceed as in $\S 3$ to solve the TOV equation and obtain $M(R)$ curves for hybrid stars. We first show a specific example, in Figure 6. We have chosen the bag model parameters to give the same equation of state for neutral matter as was studied using the phenomenological parameterization in Figure 3. Now, however, we can also study mixed phases. The solid lines, for homogeneous neutral quark and nuclear matter phases, are the same as in Figure 3. The dashed lines show the $M(R)$ relation when mixed phases are allowed. We see that although the overall shape of the $M(R)$ curve is quite different when mixed phases are present, the maximum mass is not significantly affected. The maximum mass of a compact star is therefore insensitive to the surface tension of the interface between quark matter and nuclear matter. This is because the maximum mass configuration is characterized by a baryon density that is

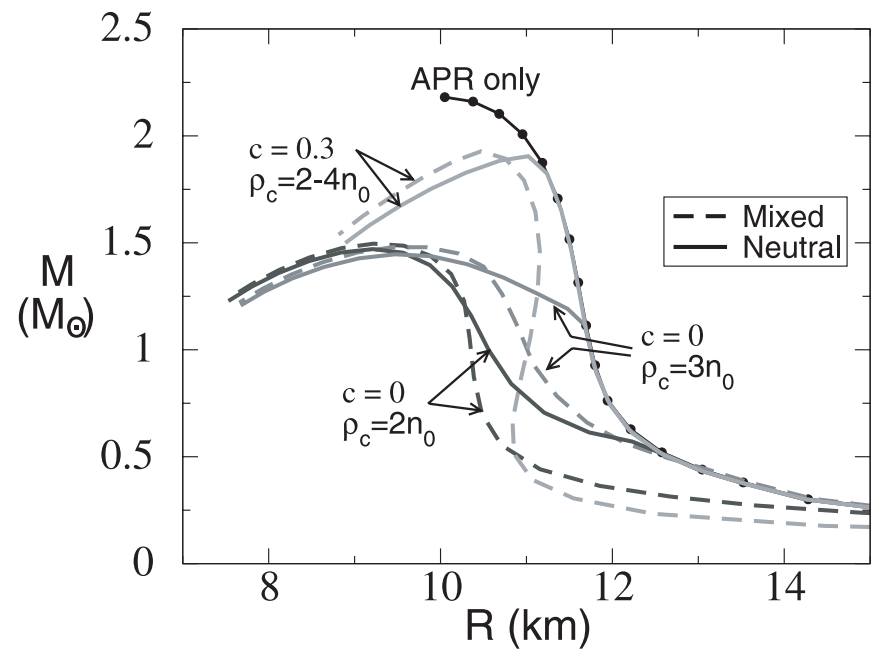

FIG. 6.-The $M(R)$ relationship for hybrid stars involving quark matter obeying the simple bag model equation of state, with various values of $c$ and $\rho_{c}$, and $m_{s}=180 \mathrm{MeV}$ and $\Delta=0$. The line with filled circles is the $M(R)$ relation for a pure nuclear APR star. The solid lines are for hybrid stars with homogeneous neutral APR and CFL phases; the dashed lines are for hybrid stars with mixed APR + CFL phases. We see that the maximum mass $M_{\max }$ is very sensitive to the perturbative correction $c$ but not to the transition density or the occurrence of mixed phases. For $c=0.3$, which is close to the value suggested in Fraga et al. (2001), $M_{\max } \approx 1.9 M_{\odot}$. [See the electronic edition of the Journal for $a$ color version of this figure.]

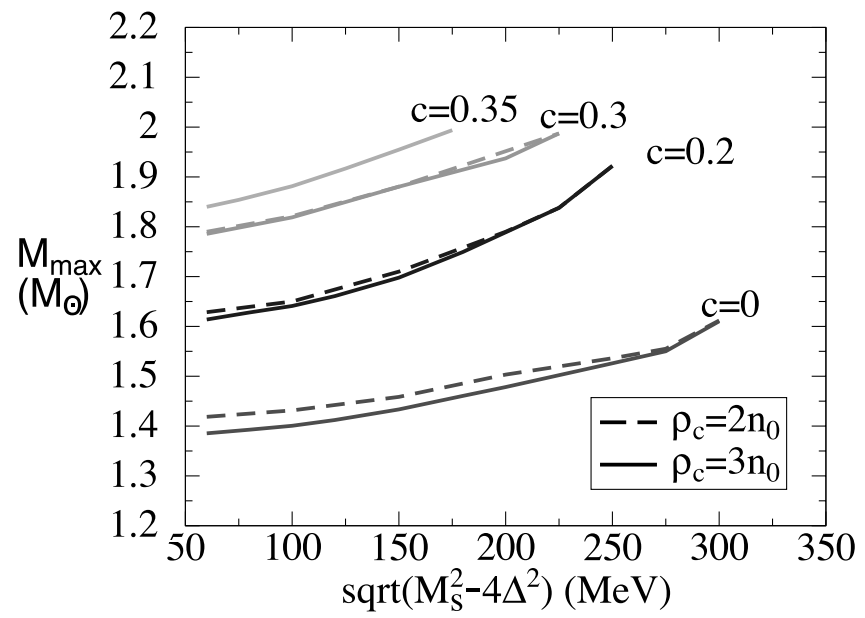

FIG. 7.-Maximum mass $M_{\max }$ for hybrid stars containing quark matter obeying the simple bag model equation of state. We show the dependence on $\left(m_{s}^{2}-4 \Delta^{2}\right)^{1 / 2}$. Increasing this quantity decreases $B_{\text {eff }}$ at fixed $\rho_{c}$, giving rise to larger maximum masses. The maximum mass is only weakly dependent on the transition density, particularly when the perturbative correction is included. [See the electronic edition of the Journal for a color version of this figure.]

large compared to the transition density and most of the star is either in the homogeneous quark matter phase or in a mixed phase that is dominated by quark matter.

We can also see that, as in the phenomenological model explored in $\S 3$, the maximum mass is very sensitive to the QCD correction $c$ and relatively insensitive to the transition density. All the curves in Figure 6 are for $m_{s}=180 \mathrm{MeV}$ and $\Delta=0$, so from this figure we cannot judge the sensitivity to those parameters.

\subsection{Maximum Mass as a Function of Bag Model Parameters}

In Figure 7 we give a more complete picture by plotting the maximum mass of the star as a function of $\left(m_{s}^{2}-4 \Delta^{2}\right)^{1 / 2}$. Varying the independent variable, $\left(4 m_{s}^{2}+\Delta^{2}\right)^{1 / 2}$, has negligible effect, as one would expect. We plot this relationship for $\rho_{c}=3 n_{\text {sat }}$ and $2 n_{\text {sat. }}$. The stars with small $m_{s}^{2}-4 \Delta^{2}$ correspond to hybrid stars containing CFL quark matter, while those with large $m_{s}^{2}-$ $4 \Delta^{2}$ correspond to stars with unpaired quark matter. We see that the largest masses are obtained by turning $m_{s}^{2}-4 \Delta^{2}$ up to a large value, corresponding to a strange quark mass in the range 200-300 MeV. This is the same situation that was described in $\S 3.3$, since the effective bag constant must decrease with increasing $m_{s}^{2}-4 \Delta^{2}$ at fixed $\rho_{c}$. As mentioned earlier, stars dominated by quark matter have $M_{\text {max }} \sim B_{\text {eff }}^{-1 / 2}$ (Witten 1984). We also see that the value of the transition density is not very important: $M_{\max }$ is only weakly dependent on the transition density, particularly when the perturbative correction is included.

\section{CONCLUSIONS}

We have studied the mass-radius relationship for hybrid compact stars, with a nuclear matter crust (described by the APR equation of state) and a quark matter core. We used a phenomenological parameterization (1) of the neutral quark matter equation of state and also a simple MIT bag model that allowed us to construct mixed phases. In both cases we included a QCD correction parameter $c$ in the quark matter equation of state, and we found that increasing its value from zero (no QCD corrections) to a reasonable value ( $c=0.35$; Fraga et al. 2001) increases the maximum hybrid star mass from about $1.6 M_{\odot}$ to about $2 M_{\odot}$. This is clear from Figure 5 (for the phenomenological parameterization) and Figure 7 (for the bag model). The reason is that increasing 
$c$ hardens the quark matter equation of state, making it almost indistinguishable from the APR nuclear equation of state (see Fig. 1). It is important to note that we achieve these masses with reasonable transition densities $\rho_{c}$ of order 2-3 times $n_{\text {sat }}$. Our stars have a proper crust of nuclear matter: we are not resorting to low transition densities that yield "hybrid" stars that are actually quark stars with a tiny shell of nuclear matter around the outside.

We conclude that it is harder than previously thought for a simple mass measurement to rule out the presence of quark matter in compact stars. Currently published measurements of the masses of compact stars are all consistent with a maximum mass of $M_{\max } \approx 1.7 M_{\odot}$ at the $2 \sigma$ level. Our results show that maximum masses of up to $2 M_{\odot}$ can be accommodated by models of hybrid stars with reasonable quark matter equations of state.

We also note that our $M(R)$ curves are consistent with the constraint obtained from measurements of red shifts of iron absorption lines in the low-mass X-ray binary EXO 0748-676 (Cottam et al. 2002). If that constraint were plotted in our Figure 3, it would intersect our $c=0.3$ curve at $M \approx 1.7 M_{\odot}$.

This naturally raises two related questions: What sort of mass or radius observation would provide evidence against the presence of quark matter in neutron stars? And what sort of observation would provide evidence for the presence of colorsuperconducting quark matter in particular?

Obviously a mass measurement above $2 M_{\odot}$ would give a reason for doubting the presence of quark matter. Other than that, it seems difficult to diagnose the presence of quark matter via $M(R)$ measurements. The regions of $M(R)$ space that can be reached by hybrid quark-nuclear stars are the same as those that can be reached by hadronic matter stars, once moderately exotic phenomena such as kaon condensation or hyperon production are allowed (Lattimer \& Prakash 2001; Haensel 2003). Observation of an object with a small radius $(R \approx 7-10 \mathrm{~km}$ at $M \approx$ 1.4 $M_{\odot}$ ) would rule out simple nucleonic matter but would not favor quark matter over the exotic forms of hadronic matter. There are regions of parameter space (very small $M$ and $R$, for example) that can only be reached by pure quark matter objects, which only exist if quark matter is absolutely stable $\left(\rho_{c}=0\right)$. Other regions of the parameter space are inconsistent with both hadronic and quark matter.

Demonstrating the presence of color-superconducting quark matter via $M(R)$ measurements appears to us to be very difficult. However, this is not for the naive reason that "the color superconductivity contribution to the pressure is suppressed by $O\left(\Delta^{2} / \mu^{2}\right)$." As previously noted (Lugones \& Horvath 2002; Alford \& Reddy 2003), the leading $\mu^{4}$ contribution is mostly canceled by the bag constant, so the subleading $\Delta^{2} \mu^{2}$ term is potentially important. However, in practice it is not detectable. First, the bag model equation of state depends on the color superconducting gap $\Delta$ via the linear combination $m_{s}^{2}-4 \Delta^{2}$, so an accurate determination of $m_{s}$ at high density would be needed to expose the presence of a nonzero $\Delta$. Second, the dependence of the $M(R)$ relation on $m_{s}^{2}-4 \Delta^{2}$ is not particularly strong (see Fig. 7) when a physical parameter $\rho_{c}$ is assumed known, rather than the bag constant.

If color-superconducting quark matter is to be found in compact stars, it seems more likely that it will be detected via its effects on transport properties. Color superconductivity drastically alters these, and possible signatures are being actively investigated. These include cooling (sensitive to heat capacity and neutrino emissivity and opacity; Reddy et al. [2003]; Jaikumar et al. [2002]; Kundu \& Reddy [2004]), $r$-mode spindown (sensitive to bulk and shear viscosity; Madsen [2000]; Manuel et al. [2004]; Drago et al. [2003]; Zheng et al. [2003]), and glitches (sensitive to superfluidity and rigid structures; Alford et al. [2001a]).

We have had useful conversations with J. Lattimer and M. Prakash. The research of M. A. and M. B. is supported by the Department of Energy (DOE) under grant DE-FG02-91ER40628. The research of $\mathrm{S}$. R. is supported by the DOE under contract W-7405-ENG-36. The research of M. P. is supported by DOE contract DE-AC05-84ER40150 under which the Southeastern Universities Research Association operates the Thomas Jefferson National Accelerator Facility.
Akmal, A., \& Pandharipande, V. R. 1997, Phys. Rev. C, 56, 2261

Akmal, A., Pandharipande, V. R., \& Ravenhall, D. G. 1998, Phys. Rev. C, 58, 1804 Alford, M. 2001, Ann. Rev. Nucl. Part. Sci., 51, 131

Alford, M., Bowers, J. A., \& Rajagopal, K. 2001a, Phys. Rev. D, 63, 074016 Alford, M., Kouvaris, C., \& Rajagopal, K. 2004, Phys. Rev. Lett., 92, 222001 2005, Phys. Rev. D., 71, 054009

Alford, M., \& Rajagopal, K. 2002, J. High Energy Phys., 06, 031

Alford, M., Rajagopal, K., Reddy, S., \& Wilczek, F. 2001b, Phys. Rev. D, 64, 074017

Alford, M., Rajagopal, K., \& Wilczek, F. 1999, Nucl. Phys. B, 537, 443

Alford, M., \& Reddy, S. 2003, Phys. Rev. D, 67, 074024

Andersen, J. O., \& Strickland, M. 2002, Phys. Rev. D, 66, 105001

Baldo, M., Bombaci, I., \& Burgio, G. F. 1997, A\&A, 328, 274

Baluni, V. 1978, Phys. Rev. D, 17, 2092

Banik, S., \& Bandyopadhyay, D. 2003, Phys. Rev. D, 67, 123003

Baym, G., Pethick, C., \& Sutherland, P. 1971, ApJ, 170, 299

Bedaque, P. F., \& Schäfer, T. 2002, Nucl. Phys. A, 697, 802

Buballa, M., Neumann, F., Oertel, M., \& Shovkovy, I. 2004, Phys. Lett. B, 595, 36

Burgio, G. F., Baldo, M., Sahu, P. K., Santra, A. B., \& Schulze, H. J. 2002a, Phys. Lett. B, 526, 19

Burgio, G. F., Baldo, M., Sahu, P. K., \& Schulze, H. J. 2002b, Phys. Rev. C, 66, 025802

Casalbuoni, R., \& Nardulli, G. 2004, Rev. Mod. Phys., 76, 263

Chodos, A., Jaffe, R. L., Johnson, K., \& Thorn, C. B. 1974, Phys. Rev. D, 10, 2599

Cottam, J., Paerels, F., \& Mendez, M. 2002, Nature, 420, 51

Drago, A., Lavagno, A., \& Pagliara, G. 2005, Phys. Rev. D, 71, 103004

Farhi, E., \& Jaffe, R. L. 1984, Phys. Rev. D, 30, 2379

Fraga, E. S., Pisarski, R. D., \& Schaffner-Bielich, J. 2001, Phys. Rev. D, 63, 121702
REFERENCES

Freedman, B. A., \& McLerran, L. D. 1977a, Phys. Rev. D, 16, 1130

- 1977b, Phys. Rev. D, 16, 1147

1977c, Phys. Rev. D, 16, 1169

1978, Phys. Rev. D, 17, 1109

Glendenning, N. K. 1992, Phys. Rev. D, 46, 1274

1997, Compact Stars, Nuclear Physics, Particle Physics, and General Relativity (New York: Springer)

Gocke, C., Blaschke, D., Khalatyan, A., \& Grigorian, H. 2001, preprint (hep-ph/ 0104183)

Grigorian, H., Blaschke, D., \& Aguilera, D. N. 2004, Phys. Rev. C, 69, 065802

Haensel, P. 2003, in Final Stages of Stellar Evolution, ed. J.-M. Hameury \& C. Motch (Les Ulis: EDP Sciences), 249

Heiselberg, H., Pethick, C. J., \& Staubo, E. F. 1993, Phys. Rev. Lett., 70, 1355 Jaikumar, P., Prakash, M., \& Schäfer, T. 2002, Phys. Rev. D, 66, 063003

Kaplan, D. B., \& Reddy, S. 2002, Phys. Rev. D, 65, 054042

Kryjevski, A., Kaplan, D. B., \& Schäfer, T. 2005, Phys. Rev. D, 71, 034004

Kundu, J., \& Reddy, S. 2004, Phys. Rev. C, 70, 055803

Lattimer, J. M., \& Prakash, M. 2001, ApJ, 550, 426 2004, Science, 304, 536

Lugones, G., \& Horvath, J. E. 2002, Phys. Rev. D, 66, 074017

Madsen, J. 2000, Phys. Rev. Lett., 85, 10

Maieron, C., Baldo, M., Burgio, G. F., \& Schulze, H. J. 2004, Phys. Rev. D, 70, 043010

Manuel, C., Dobado, A., \& Llanes-Estrada, F. J. 2004, preprint (hep-ph/0406058) Negele, J. W., \& Vautherin, D. 1973, Nucl. Phys. A, 207, 298

Nice, D. J., Splaver, E. M., \& Stairs, I. H. 2005, in ASP Conf. Ser. 328, Binary Radio Pulsars, ed. F. A. Rasio \& I. H. Stairs (San Francisco: ASP), in press (astro-ph/0411207)

Oppenheimer, J., \& Volkoff, G. 1939, Phys. Rev., 55, 374 
Pandharipande, V. R., \& Ravenhall, D. G. 1989, in Proc. NATO Advanced Research Workshop on Nuclear Matter and Heavy Ion Collisions, Les Houches, ed. M. Soyeur et al. (New York: Plenum), 103

Pandharipande, V. R., \& Wiringa, R. B. 1979, Rev. Mod. Phys., 51, 821

Pudliner, B. S., Pandharipande, V. R., Carlson, J., \& Wiringa, R. B. 1995, Phys. Rev. Lett., 74, 4396

Rajagopal, K., \& Wilczek, F. 2001, Phys. Rev. Lett., 86, 3492

2002, in At the Frontier of Particle Physics: Handbook of QCD, Vol. 3, ed. M. Shifman (Singapore: World Scientific), 2061

Reddy, S. 2002, Acta Phys. Polonica B, 33, 4101

Reddy, S., \& Rupak, G. 2005, Phys. Rev. C, 71, 025201

Reddy, S., Sadzikowski, M., \& Tachibana, M. 2003, Nucl. Phys. A, 714, 337

Rischke, D. H. 2004, Prog. Part. Nucl. Phys., 52, 197

Schäfer, T. 2003, preprint (hep-ph/0304281)
Schertler, K., Greiner, C., Schaffner-Bielich, J., \& Thoma, M. H. 2000, Nucl. Phys. A, 677, 463

Serot, B. D., \& Walecka, J. D. 1986, in Advances in Nuclear Physics, Vol. 16, ed. J. W. Negele \& E. Vogt (New York: Plenum), 1

Shovkovy, I. A. 2004, preprint (nucl-th/0410091)

Shovkovy, I., \& Huang, M. 2003, Phys. Lett. B, 564, 205

Son, D., \& Stephanov, M. 2000, Phys. Rev. D, 61, 074012 (erratum 62, 059902)

Steiner, A. W., Reddy, S., \& Prakash, M. 2002, Phys. Rev. D, 66, 094007

Thorsett, S. E., \& Chakrabarty, D. 1999, ApJ, 512, 288

Tolman, R. 1939, Phys. Rev., 55, 364

Wiringa, R. B., Stoks, V. G. J., \& Schiavilla, R. 1995, Phys. Rev. C, 51, 38 Witten, E. 1984, Phys. Rev. D, 30, 272

Zheng, X., Liu, X., Kang, M., \& Yang, S. 2004, Phys. Rev. C, 70, 015803 